\title{
Cybersport: Stages and content of usability testing of gaming interfaces and environments
}

\author{
Sergey Sergeev ${ }^{1 *}$, Arturas Kaklauskas ${ }^{2}$ \\ ${ }^{1}$ Peter the Great St. Petersburg Polytechnic University, St. Petersburg, 195251, Russia \\ 2 Vilnius Gediminas Technical University, Vilnius, LT-10223, Lithuania
}

\begin{abstract}
The article explores the content and technologies of usability testing of user aspects of gaming interfaces and elements of the gaming environment used in eSports. Areas of usability research and the specificity of the full and intermediate testing in the design of computer games are shown. The stages of preparation and testing are analyzed. The basic principles and methods of the results analysis are considered. The process of the interface design of a software product is conventionally divided into six stages. Each stage uses its own usability testing methods, and their results then become the starting point for using other methods. An enumeration and a brief overview of the research content and usability methods used at each stage of the design of the game interface and game environment are given.
\end{abstract}

\section{Introduction}

Designing digital games used in eSports is associated with the implementation of various aspects of the four main components of the game design, which include dynamic visuals, game mechanics, narration or storyline and the game play directly, which means gamer's perception of all aspects of the game in its sensory and logical unity. The ability to interact with actors and elements of the environment is provided. A gamer has a feeling of immersion in the game world, accompanied by the transformation of a cybersportman into an active player in accordance with the rules and plot of a gamer experiencing a flow of events and experience [1]. The game creation is carried out by a team of designers who ensure the fulfillment of customer requirements and decisions related to the search for palliatives and achievement of balance in the game process [2]. User properties of game program interfaces are determined using usability testing procedures, which are a set of methods and tools which allow measuring the complex characteristics of user interaction with the product [3].

Usability research of software is especially valuable when comparing specific design options for games with the aim of selecting the most effective solutions. The feedback from participants and consumers collected during usability testing is most useful when it is planned to test or improve the interaction mechanisms or the form and realization of certain elements of the game product [1].

Usability testing is particularly effective for determining:

- Textual information design of the user gaming interface and the elements of the game environment. It is possible to determine whether the instructions and orienting and suggesting elements of the interface of the program being evaluated are clear, the inscriptions on the buttons and menu bars? Perhaps some of them are perceived more easily than others?

- Architecture of the program working field. Is the information meaningfully divided into categories that do not violate the plot and logic of the game? Are the informational elements located in the places where players expect to find them?

- Accessibility of the interface for the user. Is it easy for new users to find basic interface elements? Do they understand the instructions? Do they need them?

- Efficiency of mastering the game program.

- Differentiating abilities of the game distinguishing athletes of various qualifications in order to fairly assess the results of their activities.

Usability testing focuses primarily on evaluating the first experience of product use. It is often very difficult and always time-consuming to measure the effectiveness of a solution with repeated use of a game product.

\footnotetext{
*Corresponding author: ssfpost@mail.ru
} 
Learning effects and individual differences between participants are interfering. One of the methods for overcoming these difficulties is a diary research: the subjects keep diaries with detailed records of their interaction with the product.

Finally, when conducting usability testing, you should make sure that the testing is correctly structured, that the results will be useful for identifying the problems of designing a game environment and interfaces, and you have the resources necessary to correct these problems.

During the design of user interaction with the game program, it is often desirable to assess how high-quality are the decisions that came into being. This requires offering solutions to real users. This should be done when the decision has acquired sufficient details to allow users to respond to it as to something very specific, but there is still enough time to make corrections based on the test results.

Usability tests and sessions aimed at obtaining user feedback are well suited to identify major problems with the interaction infrastructure and to improve the order and priority of actions. They are also vital for customizing user behavior. Unfortunately, it is difficult to create a test that evaluates anything other than the simplicity of a beginner learning about a solution. Methods for assessing the convenience of a product to use by the average user or expert are often used, but such methods are time consuming and give not very accurate results at best.

There are many ways to test the game interface on real users (students). These are informal feedback sessions that clarify ideas and listen to user considerations and more rigorous usability testing methods in which users solve a specific set of problems. Each approach has its advantages. More informal methods can be applied spontaneously and require less preparation. The disadvantage of this approach is that the designer is often guilty of involuntary "hints to the witness." This approach is suitable for a technical audience which is able to analyze a few drawings representing the product interface. It can be a worthy alternative to the usability test, if the design team does not have time to prepare for formal usability testing.

If there is enough time, it is necessary to give preference to more formal usability testing. Usability tests determine how well a found solution allows users to solve their problems. If the testing area is wide enough, you can also find out how well the solution helps users to achieve their goals.

Usability testing is essentially a tool for analysis, not synthesis. It does not serve as an alternative to interaction design, and will never be a source of ideas leading to the creation of an attractive product. Rather, it is a method for evaluating the effectiveness of existing ideas and a tool for their improvement.

In addition, we must bear in mind that usability testing is not a study of the user audience. Some practitioners believe that "testing" can include research (interviews, problem analysis) and even creative exercises like "user engaging design". However, this is an attempt to bring together the various needs and steps of the design process.

The study of the user audience should be conducted before the generation of ideas included in the game program, and usability testing after. It is better to spend more time making informed design decisions based on a serious research foundation than testing raw solutions without taking advantage of transparent and convenient user models, their needs and goals.

\section{Full and intermediate testing}

One distinguishes full testing when finished products are tested and intermediate testing conducted during the design process as part of the iterative process. Full testing is used to compare products, to identify problems before redesign, to investigate the causes of poor product quality and to identify requests for training and support. General studies are usually conducted by independent professionals and are documented in details.

Unfortunately, full testing is often used as part of the assessment and quality assurance process - closer to finalizing the development. At this stage, it is usually too late to make meaningful changes to the design decisions. Evaluation of the user interface should be carried out before the creation of the code begins (or, at least, early enough so that there is time to change the realization according to the additions made). However, if one wants to convince stakeholders or programmers that the problem of usability in the existing product is clearly present, then nothing will replace the observation of real users fighting with the software product.

Intermediate testing is designed just for this. These rapid qualitative tests are conducted during the design process, usually at the level of specification. Thoughtful and controlled intermediate testing allows designers to see how their target audience responds to the information and tools provided to them for solving problems.

Usability testing involves conducting experiments to identify information regarding ergonomics and user interface design. There is a common misconception that testing can solve all interface problems. With the help of testing, it is possible to identify only weak points of the interface, but it is almost impossible to detect the strong ones, since they are simply not noticed by users, and it is absolutely impossible to identify new ways to improve the interface. Usability testing is performed throughout the entire software interface development cycle. In the early stages of development, testing of a previous version or competing products makes it possible to outline test points that need to be achieved during the development process. In the middle of a project, testing provides 
feedback, telling where the interface needs improvement. In the final stages, testing verifies that the product meets (or does not fully comply with) the purposes for which it was designed.

\section{Preparation for testing}

First of all, it is necessary to put a very clear question about the purpose of testing, and in terms that allow one to unequivocally answer whether the result obtained is an answer to this question. Before testing it is necessary to clearly understand the characteristics of potential users (gamers), who should become participants of testing. This is necessary because when you will be directly involved in the selection of participants, it will be very important to know whom exactly you need.

It is necessary to determine the structure of the testing process, its composition, which describes the procedure for conducting individual tests. It is important in what order they will be built to exclude from consideration and further analysis variables that are of no interest. Tasks are being developed that will be offered to test participants. These tasks should be based on the tasks that users solve with the help of the created training product in the process of its normal use. You should specify everything you need to determine the test script: computer status, screens, documentation, help tools, and prompts that should be present. You should also indicate how the successful completion of each test task is determined.

Before testing it is also necessary to determine which auxiliary tools are needed, and purchase it. The toolkit may include instruments and devices used in the test process. These are, for example, video cameras for recording user behavior, a device for recording what is happening on monitor screens, voice recorders and audio recording equipment for recording verbal communication and verbal protocols, one-sided mirrors that allow observers and experimenters to remain invisible to test participants, and so on. You can collect a lot of useful information, using simple amateur video cameras, or even do it without video recordings [4].

It is necessary to make a list of users from which participants will be selected for each test. The number of users should be sufficient to create a representative sample, since otherwise unrecorded factors may affect the data obtained. The user profiles that you defined earlier will help you create a typical user model for your product. For example, it can be gamers of the same age group.

\section{Performance of testing}

Many people feel uncomfortable when they get into the usability lab, where they have to complete tasks, knowing that execution time is being measured, and all their errors are recorded for further analysis, so it is very difficult to make the user feel comfortable and calm. It is important to emphasize that game programs are subjected to testing, not users, and they should not feel under social pressure. Be sure to thank the users for participating in the tests. It is necessary to explain to the participants that they can stop the test at any time, or take a break if anyone needs it.

When testing, the main thing is to observe the progress of their thoughts in the process of learning the game. When there is no certainty that you understand what they are thinking, you can directly ask them about it.

The most important task of testing is to understand what expectations users have at every step, and how well the game program and its interface meet these expectations. If the user says: "I don't know what to do next," you need to ask: "What do you think, what could you do?" When the user is ready to click the mouse, you can ask him what he expects to see. After he clicks the mouse, find out if the result really met his expectation.

The difficulty is that you cannot distract users or influence them, but at the same time you need to find out what they really think (which, by the way, they themselves may not fully understand).

After each test, it is useful to make short notes about what you remember. If you do not do this before the next test, then it will be very difficult for you to remember important details.

Users often suggest solutions to a problem. Very often, such clues suggest ideas that you previously thought about, but for some reason rejected them. Sometimes it happens that some changes have been made to the project, and that method or approach that was rejected at the beginning becomes useful.

\section{Analysis of the obtained data}

After the assignments are completed and the testing is completed, the test results should be discussed with the participant. This is necessary in order to obtain additional information regarding what the user was thinking about during the test. One of the ways to analyze events is to restore them and discuss with a testing participant. In addition, you can simply ask the participant what happened during the test seemed to him worthy of attention.

After testing, each observer and assistant should write a small report on the main problems that were noticed during the testing, and state their thoughts on how to fix them, no later than the next day. Do not immediately write 
full and detailed reports. Let it be more like a summary, brief notes. Ideally, all members of the development team should read these reports (or at least run through their eyes), so the volume of these documents should not exceed 1-2 pages.

At the meeting of the working group on the results of usability research, two main issues should be discussed:

- What are the problems that users encountered when working with the tutorial program, and which ones should be fixed?

- What are the possible solutions for the selected problems?

When analyzing the data obtained, one should first look for major problems. Finding such problems is easier as they become noticeable even when viewing notes taken during user monitoring. If each participant encountered problems when using a particular menu item or solving a specific task, it is obvious that the design of this item and the task content need to be revised.

Performance data, such as error rate and task execution time, is estimated using statistical analysis methods. Most of this analysis is limited to finding the mean value and standard (root-mean-square) deviation, as well as to assessing the reliability of the differences obtained.

Observations on the actions of users and recording their self-reports both during the test (using the method of recording "thinking out loud" or asking questions) and before or after the test are carried out using check-lists and questionnaires. The structure of most of these questionnaires allows one to quantify the answers of the subjects, using a numerical scale. The obtained quantitative data can also be analyzed using standard statistical procedures for correlation and factor analysis, implemented in the SPSS, STATISTICA PRISM software packages, which are widely used in selective studies [5].

\section{Usability methods at design stages}

The practice of usability has accumulated a large number of testing methods applied to design and research of interfaces used at different usability stages. The process of the interface design of a software product is conventionally divided into 6 stages. Each stage uses its own methods, and their results then become the starting point for using other methods. There are some methods that can be applied in several stages.

1) Planning and assessment stage

At this stage, the following methods are used:

- Meeting of project participants;

- Analysis of the context of use;

- $\quad$ ISO 13407: Human centered design processes for interactive systems;

- Usability Planning;

- Analysis of competitors.

The goal of this stage is to ensure the effective use of methods during design and development processes, to ensure the constant influence of these methods on designers from the very early project stage.

At this stage, we recommend the following sequence of application of test methods:

- Start with a meeting of project participants;

- Analyze the context of use of the future product;

- Create a usability plan based on ISO 13407 ;

- $\quad$ Analyze competitors and identify strengths and weaknesses of their products.

2) The stage of drawing up requirements to the project uses the following methods:

- User survey;

- Conversations with users;

- Conversation in context;

- User monitoring/on-site research;

- Context of use;

- Focus groups;

- Brainstorm;

- Assessment of the existing system;

- Card sorting;

- $\quad$ Similarity diagrams;

- Scenarios (examples);

- Analysis of tasks;

- Collection of requirements.

At this stage, a study of users and aspects of usability is carried out, on the basis of which a specification of the requirements of the future gaming system will be compiled. The methods used at this stage are designed to collect 
information about the user interface, users, their tasks and the environment in which they are performed. The collected information is coordinated and used for the preparation of technical requirements.

Information for usability testing is collected through user surveys, conversations, conversations in a given context, user observation/research in the field, as well as using context analysis of use, focus groups, or through brainstorming and assessment the existing system.

This information is then structured using card sorting methods, affinity diagrams and developing scenarios (examples).

To study the tasks that are performed by users in the system, the method of task analysis is used.

Requirements for the created system are agreed at the meeting on the preparation of requirements. Regarding usability technical requirements for the gaming system:

- Describe the entire range of primary and secondary users of the system;

- Determine priorities for each requirement in the list based on agreed criteria;

- Determine the targets by which the usability of the created system will be assessed;

- Highlight the legal aspects of the requirements, referring to recognized standards or recommendations.

3) Design stage

- $\quad$ Design guides;

- $\quad$ Paper prototypes;

- Heuristic and expert assessment;

- $\quad$ Parallel design;

- Storyboard;

- Evaluation of the prototype;

- Templates.

4) The stage of implementation and programming

- Style rules;

- Fast prototypes.

5) Testing and assessment phase

- Diagnostic assessment;

- Performance testing;

- $\quad$ Subjective Assessment (testing \& post-release);

- Heuristic evaluation;

- $\quad$ Critical Incident Technique Analysis;

- Pleasure based approach.

6) Stage release

- $\quad$ Post release testing and measurement;

- $\quad$ Subjective Assessment (testing \& post-release);

- User surveys;

- Remote evaluation $[4,5]$.

\section{Conclusion}

Methods of usability design and usability testing are an integral part of creating gaming environments and interfaces for eSports. Phased design of all aspects of the e-sports gaming environment allows one to effectively use the entire arsenal of usability methods and technologies, improve the user properties of game interfaces.

\section{Acknowledgements}

The authors are grateful to colleagues for their help, for the special work of the technical staff on translation and editing the article. The work was performed within the implementation of the national task of the Ministry of Education and Science of Russia No. 25.8444.2017/BCh under the project "Engineering and psychological design of simulators and training systems based on symbiotic polymodal man-made environments".

\section{References}

1. N.Yu. Kazakova, Design. Materials. Technology 44 83-88 (2016)

2. B. Upton, The Aesthetic of Play (The MIT Press, Massachusetts, 2015) 
3. S.F. Sergeev, Usability testing of information systems interfaces in the humanities and art (S.-Petersburg University Publishing House, St. Petersburg, 2012)

4. A.V. Rechinsky, S.F. Sergeev, Development of user interfaces. Usability testing of information systems interfaces (Polytechnic University Publishing House, St. Petersburg, 2012)

5. S.F. Sergeev, Methods of testing and optimizing information systems interfaces (NRU ITMO, St. Petersburg 2013)

6. S.F. Sergeev, Software Engineering. 4. 42-48 (2014) 